\title{
Transposition as a function of within-pair differences'
}

LYNH OSEN AND MICHAEL COLE

UNIVERSITY OF CALIFORNIA, IRVINE

A replication and extension of Zeiler's (1966) experiment was conducted with 80 nursery school children. Experimental treatments differed in the training ratios, testing ratios and testing stimulus distances experienced by independent groups. The replication was only partially successful: (1) First test trial results showed more transposition for Ss trained with larger ratios, but no distance effect. (2) Overall test performance showed some evidence of a distance effect, but no differences attributable to the major variables. (3) A significant increase in transposition across test trials was obtained.

Zeiler (1966), in a study of the two-stimulus transposition problem, reported that four and five year old children, all of whom could verbalize the relevant relationship, transposed across all testing distances if the training problem was easy, but not if it was difficult. This finding called into question hypotheses relating the presence or absence of transposition to S's mediational capacities (e.g., Kuenne, 1946).

A weakness in Zeiler's argument is the fact that Ss who differed with respect to the ease of the training discrimination (1.4:1 vs 1.96:1 area ratios between stimuli) differed also in the ratios among testing stimuli-those with a given ratio in training experienced the same ratio among their test stimuli.

The present study replicated Zeiler's as carefully as possible with the added feature of splitting the two training groups at the time of testing into those maintaining the same ratio and those receiving the opposite ratio. The resulting experiment should permit a direct comparison of the influence of training and test ratios on transposition.

\section{Subjects}

The Ss were 96 children from 49 to 71 months of age $(\bar{X}=60.5$ months) from the Village Pre-School in Orange, California. They were assigned randomly to 16 groups of five Ss each. Fourteen Ss were dropped from the experiment for failure to reach the criterion of five successive correct choices in 45 trials. Two were dropped because of interrupted testing sessions. Apparafus

Eleven white $1 / 4$ in. Plexiglas squares served as stimuli. The squares were designated in ascending order of size as Nos. 1-11. The smallest square had a surface area of $1.19 \mathrm{sq}$. in. and each successive square increased in surface area by a factor of 1.4. The reward object was a small gold bead.

A $20 \times 24$ in. plastic-covered plyboard $1 / 2$ in. thick served as a background for displaying the stimuli. Two holes 1 in. in diameter and 8-1/2 in. apart were located in the center of the board. A $24 \times 8$ in. cardboard screen was used between trials to mask E's movements. Procedure

Two sets of training stimuli were used. For half the Ss the training stimuli were blocks No. 1 and No. 2, representing a within-set area factor of 1.4:1 (Set 1-2). For the remaining $\mathrm{Ss}$, the stimuli were blocks No. 1 and No. 3, with a within-set area factor of 1.96:1 (Set 1-3).

The $\mathrm{S}$ was instructed that the bead would be placed in the hole under one of the training blocks, and the task was to find the bead on each trial. The screen was placed between $S$ and the blocks, and the bead was placed under the larger block. The screen was removed and $S$ was asked to find the bead. A noncorrection procedure was used. The position of the larger and smaller blocks was varied randomly with the restriction that each occur equally often on each side according to a random schedule which was the same for all Ss. Criterion was five successive choices of the larger stimulus.

Once criterion was reached, testing trials were begun with no bead in the proceedings. During testing, beads were placed under both stimuli, and each $S$ was given 10 test trials. If, by the end of testing, $S$ had not spontaneously verbalized the relationship between the two blocks, E asked about the difference between the two. All Ss verbalized the relevant relationship.

\section{Experimental Design}

Up through the first transposition test trial, the experimental design is a direct replication of Zeller's, with the added subdivision of each group into those tested on the same or the opposite ratio. Thus, there are two training ratios, four testing distances (steps), and two testing ratios; when combined, these factors yield 16 groups. Because Zeller and others have found a systematic increase in transposition across testing trials, only the first test trial can yield a pure measure of transposition. Consequently, we decided to experiment with a "within-subjects" testing procedure; in testing, each $\mathrm{S}$ at a given distance experienced both training ratios five times during the testing series in a counterbalanced order. Thus, considering only test Trial 1 , we have a 2 by 4 by 2 factorial design with independent groups, but considering all test trials, we have a 2 by 4 by 2 design with all Ss receiving both levels of the third factor. Analysis for the first trial will consequently be kept separate from the analysis of the remaining trials.

\section{Results and Discussion}

Training. Analysis of variance indicated that the eight groups which received each training set did not 
differ among themselves in the trials needed to reach criterion $(F<1)$. For the groups trained with Set 1-2, the mean number of trials to criterion was 18.75 including the criterion trials; for those trained with Set $1-3$, the mean was 11.0. This difference is highly reliable $(F=19.7$, df $=1 / 72, p<.001)$. These results provide a reasonable replication of Zeiler's means, which were 19.8 and 9.28 , respectively.

Transposition Test. Table 1 shows the first test trial scores for each group. In spite of the small number of Ss in each group, the conclusion that there is no difference attributable to the size of the testing ratio seems relatively secure. Although the amount of transposition for the two training groups is not significantly different $(z=1.46, p>.10)$, the amount of transposition for the group trained with set $1-3$ is greater than chance $(z=3.48, p<.01)$ while that for the groups trained with Set $1-2$ is not $(z=1.58, p>.10)$. These results can be said to support Zeiler's findings, although there is no evidence of a distance effect and considerably less transposition overall than Zeiler observed.

An analysis of variance across test trials revealed the following: (a) transposition increased over trials ( $F=5.77, d f=4 / 288, p<.01$ ), replicating the findings of Zeiler (1966) and others; (b) there was some evidence of a distance effect $(F=2.71, d f=3 / 72, p<.10)$; (c) neither the training ratio, the testing ratio, nor any of the interactions among variables approached significance.

The large increase in transposition across test trials is illustrated in Fig. 1 for the Set 1-3 and Set 1-2 training groups.

Table 1

Transposition on test Trial 1

\begin{tabular}{|c|c|c|c|c|c|}
\hline \multicolumn{6}{|c|}{ Testing condition } \\
\hline & Steps & Set 1.3 & Set $1-2$ & Sub-total & Total \\
\hline & 1 & 5 & 3 & 8 & \\
\hline Train & 2 & 4 & 4 & 8 & 31 \\
\hline \multirow[t]{6}{*}{$1-3$} & 3 & 4 & 4 & 8 & \\
\hline & 4 & 3 & 4 & 7 & \\
\hline & 1 & 4 & 2 & 6 & \\
\hline & 2 & 3 & 4 & 7 & 25 \\
\hline & 3 & 3 & 4 & 7 & \\
\hline & 4 & 2 & $\underline{3}$ & 5 & \\
\hline Totals & & 28 & 28 & & \\
\hline
\end{tabular}

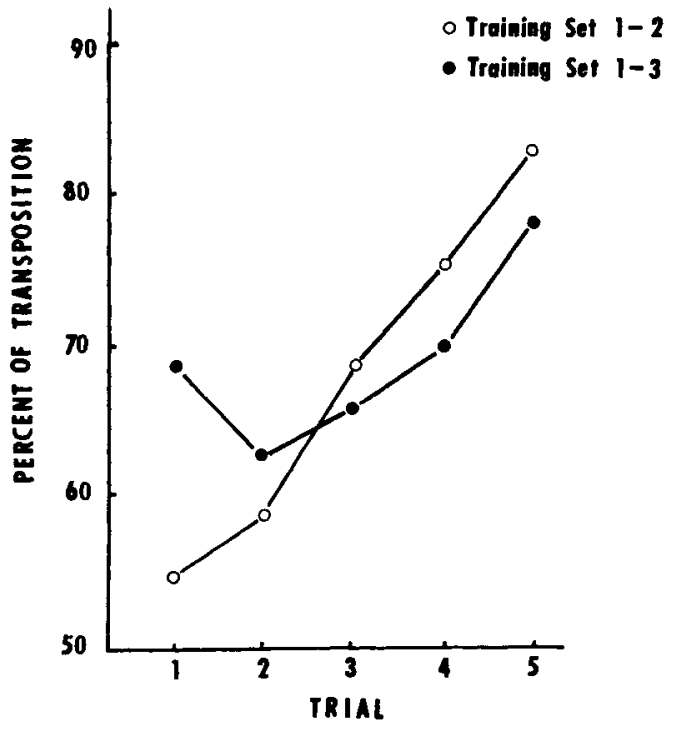

Fig. 1. The percentage of transposition responses as a function of training set and test trials.

On balance, these results indicate that the orderly picture presented by Zeiler (1966) needs further replication with sufficiently large $\mathrm{Ns}$ to make the results convincing.

The evidence obtained by Zeller (1966), Alberts \& Ehrenfreund (1951), as well as the data from the present study indicating that transposition increases over test trials in the absence of differential reinforcement receives little attention in discussions of transposition. This phenomenon, so far as we know, cannot be accounted for by extant theories of transposition, and deserves attention in its own right.

\section{References}

Alberts, E., \& Ehrenfreund, D. Transposition in children as a function of age. J. exp. Psychol., 1951, 41, 30-38.

Kuenne, M. R. Experimental investigation of the relation of language to transposition behavior in young children. $J$. exp. Psychol., 1946, 36, 471-490.

Zeiler, M. D. Solution of the two-stimulus transposition problem by four- and five-year-old children. J. exp. Psychol., 1966, 71, 576-579.

\section{Note}

1. This research reported here was part of a senior thesis conducted by the first author under the direction of the second author. Reprints may be obtained from the second author. 